\title{
Desarrollo de efectos cerámicos como acabados superficiales, mediante tecnología de inyección digital
}

\author{
FERRO SPAIN, S.A. \\ Ctra. Nacional 340, Km. 61,5. 12550 Almazora. Castellón.
}

Este trabajo ha participado en los premios Alfa de Oro en la Feria Internacional de Cerámica de Valencia CEVISAMA 2012

\begin{abstract}
Ferro Spain, S.A. ha abordado la viabilidad práctica de la decoración de superficies cerámicas mediante la aplicación de capas de muy reducido espesor mediante el uso de la tecnología de inyección digital por chorro de tinta y, específicamente en lo relativo a efectos y acabados superficiales distintos de la coloración. Se han estudiado los diversos mecanismos que permiten obtener dichos efectos y la influencia de las principales variables. También ha evaluado los resultados obtenidos atendiendo a las normativas vigentes como es el caso del efecto antideslizante.
\end{abstract}

Palabras clave: esmalte digital, inyeccion tinta, efecto ceramico digital, mate, lustre, brillo, penetrante, metalico, antideslizante.

Develop of ceramic effects: surface finishes, through digital inkject technology

Ferro Spain SA has tackled the practical viability of tile surfaces decoration by means of applying layers of reduced thickness by means of the use of digital injection technology by inkjet and, specifically, relating to effects and superficial finishes different from colouring. It has been studied several mechanisms which allow to get those effects and the influence of the main variables. It has also been assessed the obtained results dealing with the current regulations as in the case of non-slip effect.

Key words: digital glaze, inkjet, effects for digital decoration, matt, luster, glossy, sinking, metallic, anti-slippery.

\section{INTRODUCCIÓN}

La tecnología de inyección digital ha permitido en los últimos años mejorar la calidad del acabado estético de las baldosas cerámicas. De forma que con el perfeccionamiento tanto del nivel de definición como del control de la aplicación "inkjet", la traslación de la componente cromática del diseño gráfico sobre el azulejo ha alcanzado niveles considerablemente elevados.

Dentro del actual marco en el que se encuentra la tecnología de decoración digital y conociendo el grado de avance en el desarrollo de máquinas para realizar esta aplicación, según las diferentes tipologías de cabezales de inyección para la impresión "drop on demand", se plantea conseguir diferentes efectos decorativos sobre las piezas cerámicas mediante el uso de dicha tecnología, los cuales para poder ser conseguidos en la actualidad utilizan técnicas de serigrafía convencional.

La importancia del binomio definición-deposición juega un papel crítico para la obtención de los citados efectos, debido a que tanto la cantidad de material depositado como la forma y cantidad de gotas proyectadas por el cabezal de inyección, son determinantes a la hora de obtener ciertos efectos cerámicos.

\section{OBJETIVO DEL TRABAJO DE INVESTIGACION}

El presente desarrollo plantea conseguir los siguientes efectos cerámicos:

\section{Efecto mate transparente}

Aplicación de un material cerámico para conseguir efectos de maticidad, contraste y capacidad de protección mediante la superposición sobre otras superficies.

\section{Efecto brillo}

Material que permite modular la intensidad del brillo al ser aplicado sobre otras superficies, para conseguir una mayor luminosidad de la gráfica.

\section{Efecto penetrante}

Material que modifica una superficie plana para conseguir profundidad, con el fin de crear una estructura con distintos niveles.

\section{Efecto lustre}

Material que modifica la intensidad del brillo de una superficie, mediante el diferente índice de refracción entre dos vidriados, con el fin de conseguir contrastes de luz y reflejos. 


\section{Efecto metálico}

Material que cambia el acabado superficial de las baldosas cerámicas para lograr efectos de apariencia metálica.

\section{Efecto anti-deslizante}

Compuesto que altera la rugosidad superficial de la baldosa cerámica con tal de incrementar la adherencia y disminuir el deslizamiento.

En este sentido ya se había dado un primer paso en esta línea de trabajo con la obtención de un efecto "opacificante" o "blanqueante" mediante la aplicación de una tinta con tecnología de inyección digital. Este ya es un producto comercial desde principios del año 2011.

El trabajo tiene como objetivo pues, el desarrollo de nuevas composiciones de tintas inkjet para lograr los efectos anteriormente detallados, con las limitaciones que presenta la tecnología actual asociada a los inyectores que depositan la tinta sobre las piezas cerámicas. En concreto, las citadas limitaciones son: el tamaño máximo de partícula aceptable según la actual tecnología de cabezales de impresión así como la cantidad máxima de tinta que se puede depositar según la velocidad de la línea de esmaltado.

Para la consecución del objetivo del desarrollo planteado se siguen los siguientes pasos:

- Estudiar de qué forma la limitación del tamaño de partícula del material depositado influye en la obtención de los efectos deseados. Al mismo tiempo es necesario conocer el mecanismo por el cual cristalizan las diferentes fases cristalinas, de distinto índice de refracción, que influyen en la obtención de los diferentes efectos.

- Desarrollar los procesos específicos tanto a nivel de laboratorio como a escala semi-industrial para la obtención de las diferentes tintas con las que poder aplicar en máquinas de decoración digital.

\section{DESARROLLO EXPERIMENTAL}

Durante la etapa de estudio y desarrollo del nuevo conjunto de tintas, se analizaron las diferentes propiedades y características técnicas necesarias para obtener los diferentes efectos cerámicos deseados, particularmente, qué requisitos teóricos son necesarios establecer para conseguir los objetivos marcados. A continuación se detallan las principales características de cada una de las tintas obtenidas.

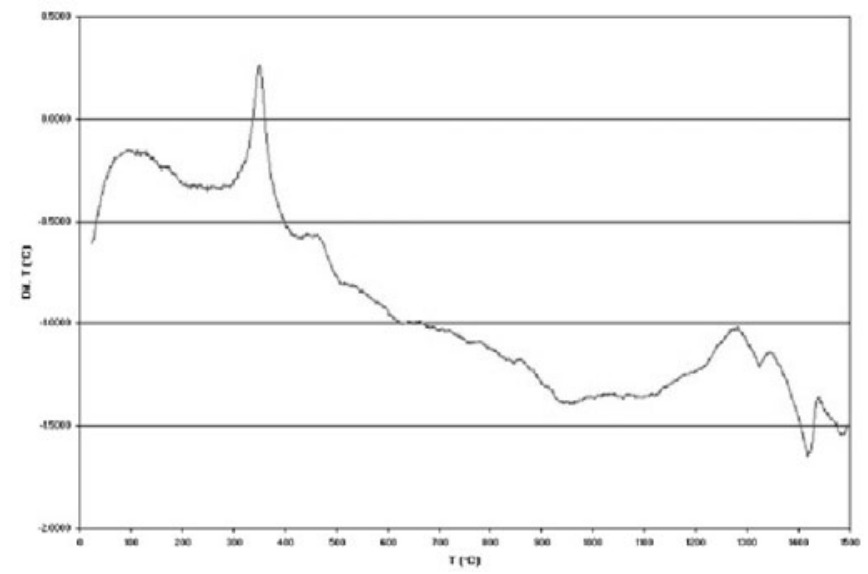

Fig. Análisis Térmico Diferencial (ATD) de la frita de alto tránsito.

\section{Tinta efecto mate}

Se ha desarrollado una nueva frita mate de alto punto de reblandecimiento que evita ser modificada su estructura cristalina por los esmaltes actuales, por lo que se consigue que la tinta con este efecto, se mantenga "flotando" obteniéndose un efecto de profundidad sobre la superficie vítrea.

Dicha frita es capaz de desvitrificar una alta proporción de cristales de Cordierita que le confiere una alta resistencia al tránsito y resistencia a ensayos físico-químicos.

Sólo la capa muy fina aplicada con tecnología inkjet, de este tipo de frita es capaz de proporcionar este acabado cerámico con unas propiedades técnicas excepcionales.

\section{Tinta efecto brillo}

Dado los buenos resultados obtenidos con la tinta de efecto mate, se decidió desarrollar una tinta que presentara un efecto opuesto al mate y que está muy extendido como es el efecto brillo.

Para el desarrollo de esta tinta se ha buscado una frita cuya superficie tuviera un alto brillo (alta capacidad de reflejar la luz) y presentar un bajo punto de reblandecimiento ya que las condiciones a las que se iba a aplicar la tinta con efecto brillante no son las óptimas debido a que la capa es muy fina.

Adicionalmente con estas propiedades se pretende conseguir que la superficie de la capa aplicada presente una buena estirada y no presente defectos superficiales, es decir tenga un buen poder cubriente.
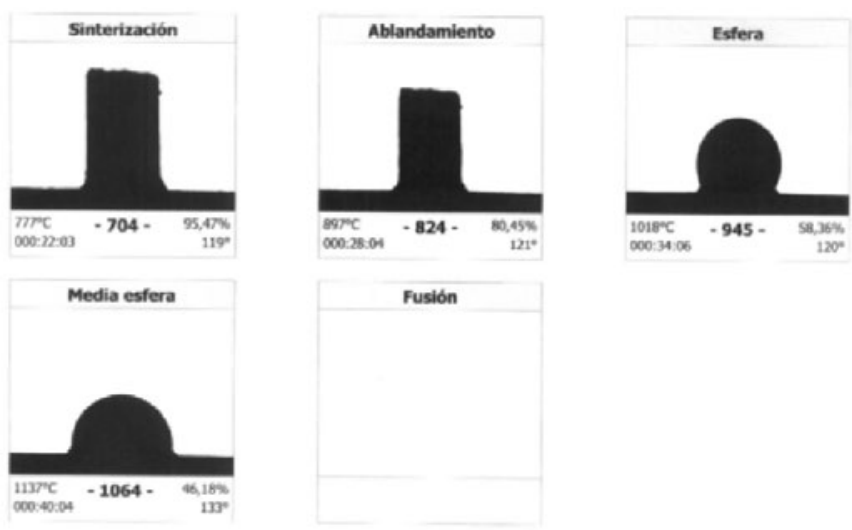

Fig. Microscopía de calentamiento de la frita de alto brillo.

\section{Tinta efecto Anti-Slip}

Además de buscar una cuidada estética, en los últimos años se ha perseguido con especial interés el ofrecer un mayor valor añadido a través del concepto de funcionalidades especiales, como por ejemplo que sean resistentes al deslizamiento.

Por ello, se ha desarrollado una tinta, cuyo objetivo principal es obtener unos valores de resistencia al deslizamiento elevados manteniendo una superficie suave al tacto.

Dicha aplicación tiene que ser "invisible", es decir transparente y que no altere el aspecto del azulejo. Este hecho se consigue mediante la combinación del tipo de grafica aplicada y la cantidad de material depositado.

La estructura cristalina de esta tinta está basada en una combinación de a-Alúmina altamente sinterizada con una frita compatible capaz de unirse fuertemente a las partículas de aquella y obtener resistencia química, transparencia y al 


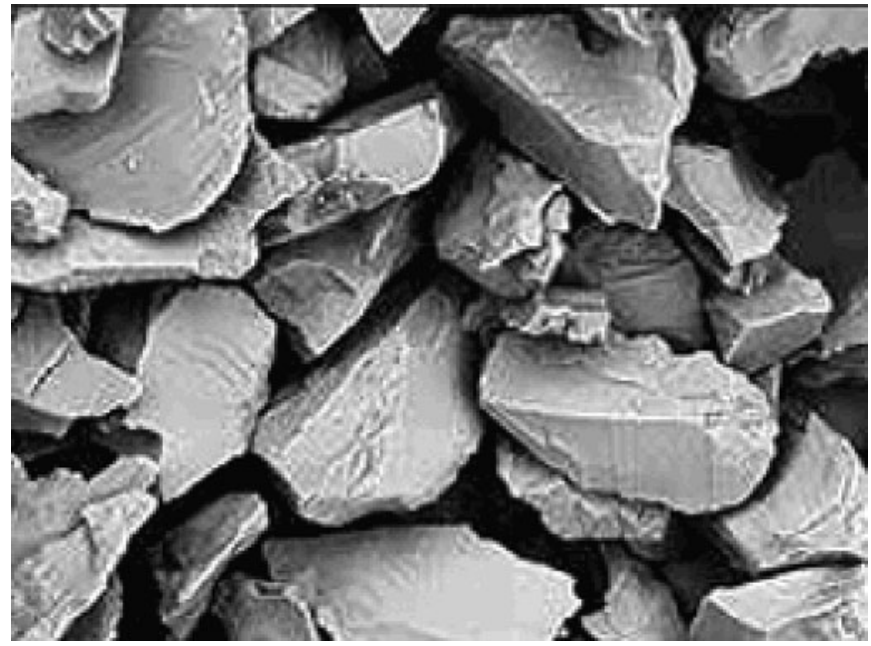

Fig. Micrografía de Microscopía Electrónica de Barrido de las partículas de la tinta con efecto Anti-slip.

tiempo que se alcanzan elevados valores de resistencia al deslizamiento, determinada mediante el ensayo del péndulo descrito en el Anexo A de la norma UNE-ENV 12633:2003.

\section{Efecto Lustre}

En el conjunto de efectos cerámicos, uno de los que tiene una relevante importancia por el aporte estético que realiza sobre los diseños, es el reflejo lustre. Este efecto consiste en ser capaz de devolver la mayor parte de energía lumínica que recibe.

El objetivo era conseguir una superficie lustre, transparente y que respetara el desarrollo del color del resto de tintas. Siendo que las actuales serigrafías lustre tienden a opacificar y modifican seriamente el desarrollo del color de las tintas. A parte de necesitar mayores cantidades de material depositado para obtener el efecto lustre deseado.

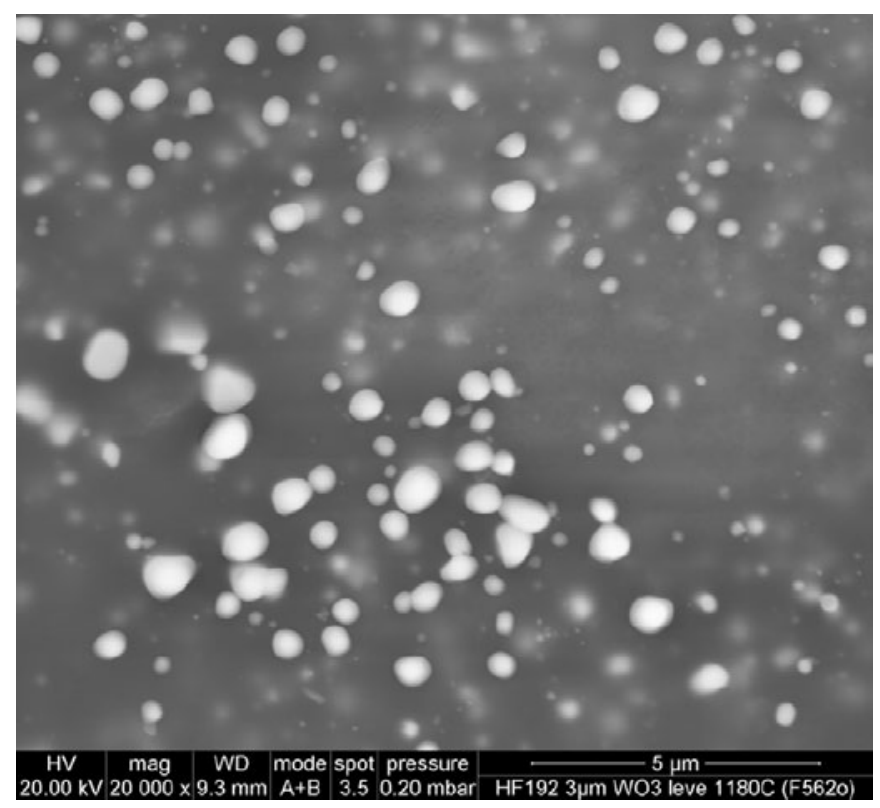

Fig. Micrografía de Microscopía Electrónica de Barrido mostrando las partículas de scheelita (puntos brillantes).
Además, dada la actual problemática que existe con las materias primas, entre ellas el óxido de cerio, la nueva tinta con efecto lustre se posiciona como una alternativa importante ya que dejaríamos de depender del óxido de cerio.

La alternativa al cerio es el wolframio, el compuesto de wolframio se aplica en una capa muy fina que en cocción reacciona con el esmalte dando una cristalización concreta orientada y estable. Dicha cristalización tiene lugar gracias a la formación de cristales de scheelita cuyo efecto lustre es adamantino.

\section{Tinta Efecto Penetrante}

Una de las serigrafías de gran uso y conocidas desde los inicios en cerámica es el efecto penetrante, dado que la mayoría de nuestros clientes tienen un consumo de este tipo de productos, obtener dicho efecto mediante una aplicación con inyección era muy interesante. Por ello, el objetivo era alcanzar un grado de hundimiento en el esmalte similar al que se dispone con las serigrafías penetrantes actuales. Otra de las características es que fuera transparente y no peligroso para el medio ambiente ya que esta empresa tiene entre sus principales objetivos el de desarrollar productos que no dañen el medio ambiente.

El efecto de penetración en el esmalte de este tipo de tinta viene determinado por su baja viscosidad y tensión superficial en fundido.

Normalmente las serigrafías penetrantes tradicionales necesitan una capa importante para poder alcanzar este tipo de efecto, mientras que con esta composición en forma de tinta se consigue el efecto deseado con una cantidad de material depositado muy inferior.

\section{Tinta Efecto Metálico}

Dada la demanda del efecto metálico como elemento decorativo en el sector cerámico, en nuestros laboratorios se ha obtenido la posibilidad de aplicar dicho efecto mediante la tecnología de inyección digital. Dado que de esta forma nos permite poder decorar piezas en zonas muy concretas y con una alta definición.

Las ventajas de esta aplicación son: precisión y control de la capa de tinta que se deposita sobre la pieza por lo que se aumentan las posibilidades de decoración y se elimina el problema de tonos que suele aparecer cuando se trabaja con metálicos.

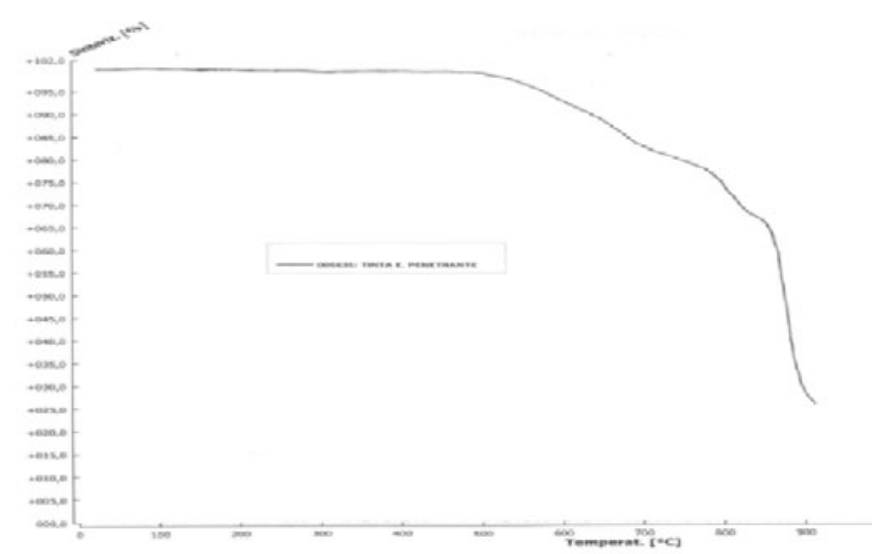

Fig. Curva de Sinterización de la Tinta Efecto Penetrante. 
Es necesaria la aplicación de dos capas distintas de material cerámico para que reaccionen entre sí para obtener efecto lustre metalizado.

La primera capa se trata de una frita especial que actúa como precursora de la orientación de los cristales que se generan en la segunda capa (superficie) que dan como resultado el efecto metálico.

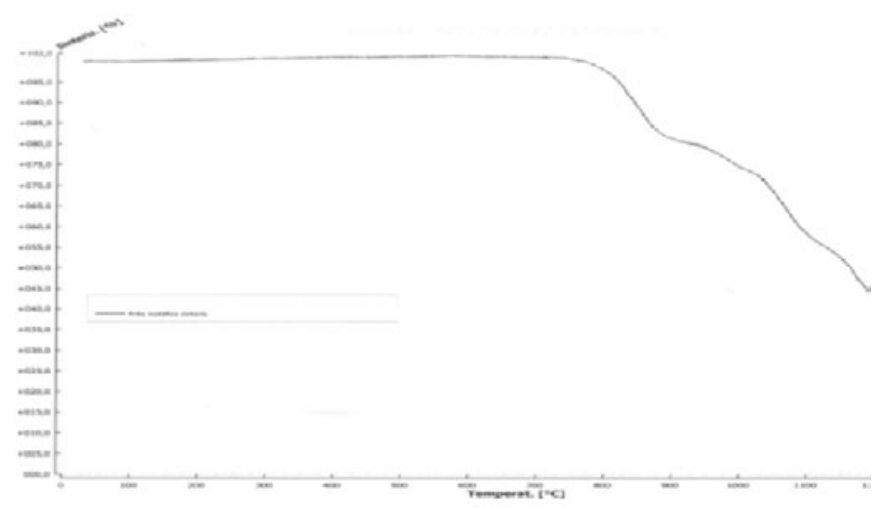

Fig. Curva de Sinterización de la Frita que metaliza.

\section{CONCLUSIONES}

Se ha demostrado la viabilidad práctica, con procesos e instalaciones actuales, de la obtención de una serie de efectos estéticos y funcionales aplicando capas muy delgadas utilizando la tecnología digital de inyección de chorro de tinta.

Entre los diversos mecanismos que permiten obtener estos efectos, destaca el de la formación de una capa uniforme de scheelita sobre la superficie del vidriado generando una diferencia neta de índice de refracción y permitiendo de este modo la difracción diferida que se observa como efecto lustre por el observador.

Las variables más relevantes son la definición, volumen de deposición, distribución de tamaño de partícula, naturaleza de las partículas, reología, temperatura de reblandecimiento e índice de refracción entre otras.

Fotografías de las piezas cerámicas con los distintos efectos conseguidos:

\section{BIBLIOGRAFÍA}

1. G. Cao. Nanostructures \& Nanomaterials. Synthesis, Properties \& Applications. World Scientific. Singapore, 2006

2. K.G. Ewsuk, Y. Gogotsi. Characterization, Design, and Processing of Nanosized Powders and Nanostructured Materials. Ceramic Transactions Vol. 190. Wiley. New Yersey, 2006.

3. M. Rhodes. Introduction to Particle Technology. Wiley, 2nd edition. Chichister, 2008

4. R. W. Balluffi, S.M. Allen, W.C. Carter. Kinetics of Materials. Wiley. New Yersey, 2005.

5. K. Sangwal. Additives and Crystallization Processes. From Fundamentals to Applications. Wiley. Chichister, 2007.

6. D. Brandon, W.D. Kaplan. Microstructutal Characterization of Materials. Wiley, $2^{\text {nd }}$ edition. Chichister, 2008.

7. T.F. Tadros. Rheology of Dispersions. Principles and Applications. Wiley-VCH. Weinheim, 2010.

8. A.R. West. Solid State Chemistry and its Applications. Wiley, $1^{\text {st }}$ edition. 1987.

9. J.M . Rincón, M. Romero. Characterization Techniques of Glasses and Ceramics. Springer. Heidelberg, 1999.

10. J. Goldstein, D. Newbuty, D. Joy, C. Lyman, P. Echlin, E. Lifshin, L. Sawyer, J. Michael. Scanning Electron Microscopy and X-Ray Microanalysis. Kluwer Academic, $3^{\text {rd }}$ edition. New York, 2003.

11. S. Magdassi. The Chemistry of Inkjet Inks. World Scientific. Singapore, 2010.

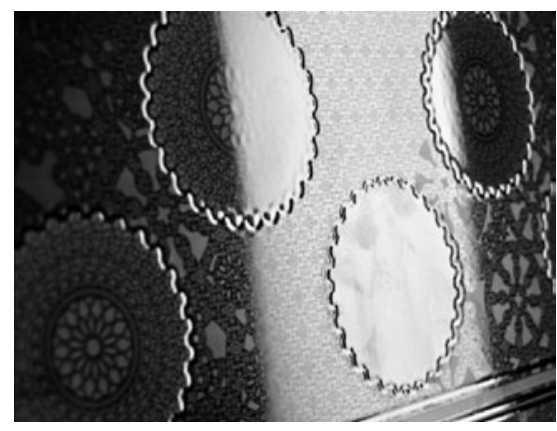

Efecto mate, penetrante y blanca.

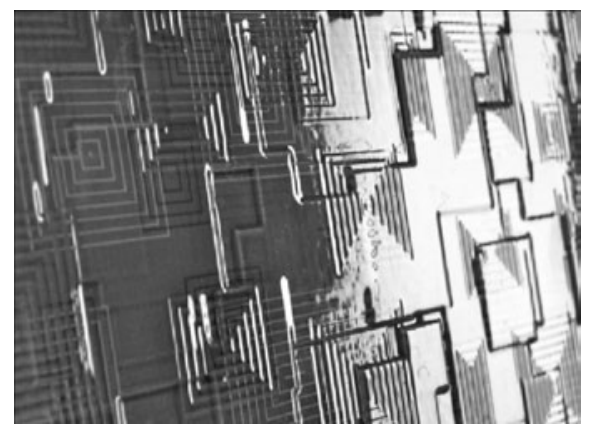

Efecto mate, penetrante y blanca.

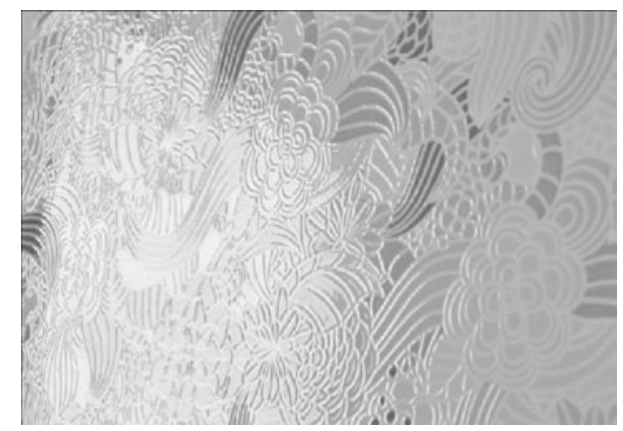

Efecto mate, pentrante y blanca. 